\title{
Participación de las mujeres en la carrera de Ingeniería Informática de la Universidad Nacional y su desempeño en los cursos de programación
}

\author{
Women's Participation in the Information Systems Career at the National University of \\ Costa Rica and Their Performance in Programming Courses
}

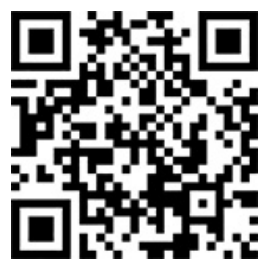

\author{
Sonia Mora-Rivera ${ }^{1}$ \\ Universidad Nacional \\ Heredia, Costa Rica \\ sonia.mora.rivera@una.cr \\ http://orcid.org/0000-0002-8333-684X \\ Mayela Coto-Chotto ${ }^{2}$ \\ Universidad Nacional \\ Heredia, Costa Rica \\ mcoto@una.cr \\ http://orcid.org/0000-0002-4558-3671

Johnny Villalobos-Murillo
Universidad Nacional
Heredia, Costa Rica
jvillalobos@una.cr

Recibido 18 de enero de 2016 • Corregido 28 de octubre de 2016 • Aceptado 29 de noviembre de 2016

\begin{abstract}
${ }^{1}$ Tiene una licenciatura en Ciencias de la Computación e Informática de la Universidad de Costa Rica y una maestría en Tecnología e Informática Educativa de la Universidad Nacional. Actualmente es catedrática en la Escuela de Informática de la Universidad Nacional. Sus áreas de especialidad comprenden el desarrollo de aplicaciones tanto comerciales como educativas, y la aplicación de nuevos enfoques pedagógicos y metodológicos aplicados al área de la Ingeniería Informática. En los últimos 15 años ha realizado investigaciones en enfoques pedagógicos, espacios de aprendizaje virtuales, el uso de recursos educativos abiertos (REA) y los ambientes de aprendizaje colaborativos y ubicuos (u-CSCL). Cuenta con 30 años de experiencia en educación superior como directora, subdirectora, coordinadora académica, profesora y coordinadora en cursos de grado de las carreras de Ingeniería de Sistemas de Información e Informática Educativa. Posee publicaciones en libros, revistas y conferencias nacionales e internacionales.

${ }^{2}$ Tiene una licenciatura en Ciencias de la Computación e Informática de la Universidad de Costa Rica, una maestría en Ciencias de la Computación del Instituto Tecnológico de Costa Rica y un doctorado en Human Communication Centered Informatics de la Universidad de Aalborg en Dinamarca. Actualmente catedrática en la Escuela de Informática de la Universidad Nacional. Sus áreas de especialidad comprenden el desarrollo de propuestas sobre la implementación de tecnologías de información y comunicación en diferentes niveles y contextos educativos. Específicamente el desarrollo de software educativo, uso no tradicional de espacios virtuales, y nuevos enfoques pedagógicos y metodológicos aplicados al área de la ingeniería informática. Ha realizado investigación en el uso de comunidades de práctica (CoP) como un medio de desarrollo profesional docente en el área TIC, el uso de recursos educativos abiertos (REA) y los ambientes de aprendizaje colaborativos y ubicuos (u-CSCL). Cuenta con 29 años de experiencia en educación superior como profesora en cursos de grado y de posgrado de las carreras de Ingeniería de Sistemas e Informática Educativa, además de la participación en la supervisión de tesis de maestría. Posee múltiples publicaciones en libros, revistas y conferencias nacionales e internacionales.

${ }^{3}$ Tiene una licenciatura en Informática de la Universidad Nacional de Costa Rica y una maestría en Ciencias de la computación y Auditoría de Tecnologías de Información de la Universidad de Costa Rica. Sus áreas de especialización se enfocan en la inteligencia de negocios, análisis de datos y minería de datos así como en la seguridad de la información. Cuenta con más de 25 años de experiencia en la educación superior como profesor de grado y posgrado en las carreras de Ingeniería de Sistemas e Informática. Posee múltiples publicaciones en revistas y conferencias a nivel nacional e internacional. Actualmente es profesor e investigador en la Universidad Nacional de Costa Rica y de la Universidad de Costa Rica.
\end{abstract}


doi: http://dx.doi.org/10.15359/ree.21-1.12

URL: http://www.una.ac.cr/educare

CORREO: educare@una.cr

Resumen: Ha sido bien documentada a nivel mundial la baja presencia de mujeres en las carreras de informática. El presente artículo describe la situación actual en la Carrera de Ingeniería en Sistemas de Información de la Escuela de Informática de la Universidad Nacional de Costa Rica (UNA). El problema de la baja participación femenina se analiza en dos dimensiones, el reclutamiento y la retención. Para estudiar el reclutamiento se analizaron los índices institucionales de postulación y admisión a la carrera. Para estudiar la retención, se analizó el desempeño del estudiantado en el área de programación por ser considerada esta como un área central en el programa de estudios y como un área de dificultad para el estudiantado. El estudio fue realizado con los datos obtenidos del Departamento de Registro de la UNA durante un período de 8 años, del año 2007 al año 2014. Los hallazgos coinciden con los resultados a nivel mundial en este tema y los patrones de permanencia son similares para ambos sexos. Con respecto al tiempo de graduación las mujeres tienden a ser más efectivas, es decir, sus tiempos de graduación son menores. Los dos primeros cursos de programación presentan gran dificultad para la mayoría de estudiantes, pero las mujeres tienden a tener menor desempeño. En general, este estudio pretende ser un punto de partida para plantear acciones educativas que permitan mejorar los índices de ingreso y permanencia de las mujeres en la carrera $y$, a la vez, aumentar el número de personas graduadas en el área, asunto que es de interés institucional, nacional e internacional.

Palabras claves: Admisión académica; educación superior; mujeres; ingeniería; programación; informática.

\begin{abstract}
It is well documented worldwide the low presence of women in informatics careers, and this phenomenon seems to have grown in recent years. This article describes the current situation in the Information Systems career in the School of Informatics at the National University of Costa Rica (UNA). The problem of low female participation is analyzed in two dimensions: recruitment and retention. To study recruitment, we have analyzed the institutional indices of application and admission. To study retention, we have analyzed student achievement in the programming area, because it is considered a central area in the curriculum and an area of difficulty for students. The analysis is performed using data obtained from the UNA Registration Department in a period of 8 years, from 2007 to 2014. The findings support the worldwide results about the underrepresentation of women in the career. Permanency patterns are similar for both sex, and women tend to be more effective in graduating. Additionally, the first two courses of programming seem to represent difficulties for all students, but women tend to have lower performance. In general, the study aims to be a starting point to propose educational actions to improve the rates of representation and permanence of women in the career, while increasing the number of graduates in the area; an issue that is of institutional, national and international interest.
\end{abstract}

Keywords: Academic admission; higher education; women; engineering; computer programming.

\title{
Introducción
}

Numerosos estudios han identificado la baja participación de las mujeres en carreras de informática y computación (Beyer, Rynes, Perrault, Hay y Haller, 2003; Katz, Allbritton, Aronis, Wilson y Soffa, 2006; Klawe y Leveson, 1995; Margolis y Fisher, 2003; Vilner y Zur, 2006). Debido a este fenómeno de carácter mundial y a la creciente necesidad de profesionales en esta área, resulta fundamental plantearse: ¿por qué la disciplina resulta tan poco atractiva para las mujeres?, y ¿de qué manera pueden las instituciones interesadas abordar esta situación? 
doi: http://dx.doi.org/10.15359/ree.21-1.12

URL: http://www.una.ac.cr/educare

CORREO: educare@una.cr

Se ha determinado que hay un número de factores que disuaden a las mujeres de cursar carreras de informática, entre ellos, la imagen de la informática como un dominio masculino; la falta de confianza entre las estudiantes a pesar de sus obvias habilidades; la falta de mujeres docentes y modelos a seguir; una cultura que no invita a las mujeres a incursionar en la informática y la importancia de la experiencia previa en programación (Fisher, Margolis y Miller, 1997; Lewis, Lang y McKay, 2007; Margolis y Fisher, 2003; Miliszewska, Barker, Henderson y Sztendur, 2006; Scragg y Smith, 1998; Stoilescu y Egodawatte, 2010; Wilson, 2002). Las investigaciones demuestran que no hay una respuesta sencilla al hecho de que las mujeres no se decidan más por estudiar carreras de informática, ya que las razones parecen estar vinculadas con la naturaleza de los roles socialmente definidos. Sin embargo, según Byrne y Lyons (2001), entender la situación de las mujeres en sus estudios puede brindar algunos indicios que permitan entender cómo apoyar sus esfuerzos y como mantenerlas dentro de las carreras de informática.

En este contexto, este artículo analiza el ingreso y permanencia de hombres y mujeres en la carrera de Ingeniería en Sistemas de Información de la Escuela de Informática de la Universidad Nacional. Adicionalmente, se estudia la situación de ambas poblaciones en los cursos de programación del plan de estudios, con el fin de determinar si esta área tiene algún impacto en la permanencia o no de las mujeres en la carrera. Dado que el género es una construcción simbólica creada por la sociedad y no relacionada con los datos biológicos al nacer (Lamas, 1997), este estudio no puede considerarse un análisis de género.

El objetivo de la investigación es identificar el porcentaje de participación de las mujeres en la carrera de Ingeniería en Sistemas en Información y su desempeño académico en los cursos de programación. El estudio es relevante, ya que la baja representación femenina en dicha disciplina puede tener un impacto negativo en una sociedad que es cada vez más dependiente de la tecnología. De continuar con este ritmo decreciente, la sociedad va a ir perdiendo la presencia de las mujeres en los ámbitos de producción, innovación, transferencia y desarrollo del conocimiento científico. Los resultados obtenidos pueden posteriormente derivar en algunas estrategias que permitan ir incrementando la participación femenina en la carrera, tanto a nivel estudiantil como a nivel docente.

El artículo plantea las siguientes interrogantes:

1. ¿Cuál es la representación de la mujer en la carrera de Ingeniería en Sistemas de Información de la UNA?

2. ¿Cuáles son los índices de aprobación en el área de programación? ¿Muestran alguna diferencia los resultados de las mujeres con respecto a los hombres?

3. ¿Qué impacto tienen los dos primeros cursos de programación en la permanencia de las mujeres en la carrera? 
doi: http://dx.doi.org/10.15359/ree.21-1.12

URL: http://www.una.ac.cr/educare

CORREO: educare@una.cr

El artículo tiene la siguiente estructura: inicialmente se describe el fundamento teórico que da pie a la investigación propuesta, incluyendo el área específica de programación y el contexto nacional; en la siguiente sección se describe la metodología seguida en el procesamiento de los datos; posteriormente se analizan los datos desde dos dimensiones: el reclutamiento, es decir, el proceso mediante el cual el estudiantado es admitido a la carrera de Ingeniería en Sistemas de Información y la retención o permanencia en la carrera; finalmente se presentan las conclusiones y se esboza el trabajo futuro.

\section{Fundamento teórico}

Wilson (2002) indica que el problema de la baja participación femenina en elárea informática se da en dos dimensiones: en el reclutamiento y en la retención. Es decir, hay pocas mujeres que se matriculan en las carreras de informática y una vez matriculadas es difícil mantenerlas en la carrera. Agrega que esta tendencia a la disminución de mujeres que completan sus estudios universitarios en informática también repercute posteriormente en el profesorado y en su trayectoria académica, por ejemplo, en el paso de categoría de profesora instructor a profesora asistente y de esta a profesora catedrática, lo que crea una importante brecha en esta disciplina.

Con respecto al reclutamiento, se han hecho numerosos estudios (Fisher et al., 1997; Margolis y Fisher, 2003; Scragg y Smith, 1998), que muestran que las diferencias en la participación de las mujeres, con respecto a los hombres, en la informática no se debe a diferencias de habilidad o capacidad de estas, sino que está ligada a factores como la falta de modelos y de apoyo, los estereotipos, y una percepción de falta de autoestima y capacidad entre las mujeres (Lewis et al., 2007; Miliszewska et al., 2006; Stoilescu y Egodawatte, 2010).

Con respecto a la retención, los estudios para explicar la alta deserción de mujeres en programas de informática se han enfocado en factores como: la experiencia previa con computadoras (Byrne y Lyons, 2001; Hagan y Markham, 2000; Lewis et al., 2007; Wilson y Shrock, 2001), un ambiente y una cultura hostil, la teoría de la atribución y la creencia de autoeficacia (Margolis y Fisher, 2003; Sackrowitz y Parelius, 1996; Scragg y Smith, 1998). La teoría de la atribución sugiere que cuando las personas atribuyen sus éxitos a causas inestables (suerte o esfuerzo) y sus fracasos a causas estables (habilidad o dificultad de la tarea), la probabilidad de persistencia es baja. La creencia de auto-eficacia se define como la valoración que realiza el individuo de sus propias habilidades para ser exitoso en una actividad específica. En el caso de las mujeres en informática, se ha determinado que las mujeres, al contrario que los hombres, tienden a atribuir su "éxito" a la suerte y su "fracaso" a la falta de capacidad (Wilson, 2002). Se ha determinado, también, que la baja confianza que las mujeres tienen en su capacidad de trabajo con las computadoras es una de las principales barreras para su ingreso y avance en la disciplina (Beyer et al., 2003; Lewis et al., 2007). 
doi: http://dx.doi.org/10.15359/ree.21-1.12

URL: http://www.una.ac.cr/educare

CORREO: educare@una.cr

Adicionalmente, otros estudios argumentan que los cursos de informática tienen una reputación de ser aburridos y de no brindar al estudiantado libertad creativa, lo que desalienta a las mujeres a entrar en el campo (Rich, Perry y Guzdial, 2004). Además, ellas tienen, antes de ingresar a la universidad, menos experiencia con la informática que la mayoría de los hombres, ya que estos experimentan un entusiasmo por los computadores mientras que son muy jóvenes, y las mujeres despiertan este interés en una etapa posterior (Beyer et al., 2003). Asimismo, esta falta de experiencia ocasiona que tengan un punto de partida desventajoso, lo que hace que se sientan inferiores y menos confiadas que los hombres (Margolis y Fisher, 2003). Por otro lado, se dice que las mujeres no reciben el mismo nivel de apoyo que los hombres para entrar y permanecer en la carrera (Cohoon, 2002).

\section{La perspectiva en programación}

Se ha considerado que la experiencia previa en programación es un elemento predictor del éxito en los cursos de programación (Hagan \& Markham, 2000; Wilson y Shrock, 2001). Algunas investigaciones (Margolis y Fisher, 2003; Sackrowitz y Parelius, 1996; Scragg y Smith, 1998) han documentado que las mujeres ingresan a las carreras universitarias de informática con menos experiencia previa en programación que los hombres y que, además, estas tienen más ansiedad y menos confianza en sus habilidades de computación (Beyer, Dekeuster, Walter, Colar y Holcomb, 2005; Beyer et al., 2003; Margolis y Fisher, 2003; Scragg y Smith, 1998; Stoilescu y Egodawatte, 2010). Para Beyer et al. (2003), esto puede explicarse por el hecho de que las mujeres desarrollan el interés en la programación a una edad más avanzada que los hombres. Esta falta de experiencia en programación es probable que afecte negativamente la confianza de las mujeres, ya que una percepción positiva de esta habilidad está ligada directamente a la motivación intrínseca y a la persistencia, y una percepción negativa puede disminuir la probabilidad de que las mujeres opten por la disciplina informática y aumenta la probabilidad de que abandonen la carrera, con lo cual pierden la oportunidad de entrar en un campo muy bien pagado y con un excelente potencial de desarrollo profesional.

Otros estudios no han encontrado evidencia para argumentar que el conocimiento previo en el área de programación sea un factor de éxito (Byrne y Lyons, 2001; Murphy et al., 2006; Ventura y Ramamurthy, 2004). Murphy et al. (2006) no encontraron una correlación entre la experiencia anterior de estudiantes en programación y el éxito final en los estudios, medido este como el promedio de calificaciones obtenido al graduarse. Los datos también indicaron que una vez superados los cursos introductorios, las mujeres muestran un nivel de dominio de los conceptos de programación casi igual al de los hombres. La explicación que brindan para esta situación es que la mayoría de la experiencia previa que tienen los hombres es en enfoques de programación no orientados a objetos, por lo que en realidad no proporcionan una ventaja cuando se trata de cursos de programación introductorios que utilizan un enfoque orientado 
doi: http://dx.doi.org/10.15359/ree.21-1.12

URL: http://www.una.ac.cr/educare

CORREO: educare@una.cr

a objetos. En la misma línea, Ventura y Ramamurthy (2004) indican que la experiencia previa en programación no es un predictor de éxito para cursos introductorios de programación orientados a objetos.

Vilner y Zur (2006) han identificado que los cursos que ofrecen más dificultades a las mujeres son los primeros cursos en el programa de estudios tales como Introducción a la Informática/Computación, Cálculo y Álgebra Lineal, y los cursos más prácticos como Diseño Digital, Redes de Computadoras y Organización de Computadoras. Ramírez y Rodríguez (2013) mencionan específicamente la dificultad de los cursos de programación y su incidencia en la disminución de las mujeres. Mientras que otros estudios muestran que el sexo afecta únicamente el rendimiento en el primer curso de programación (Beyer et al., 2005; Goold y Rimmer, 2000), y que a partir de este punto no hay ninguna diferencia significativa entre el rendimiento de los estudiantes masculinos y femeninos (Byrne y Lyons, 2001). En general, hay coincidencia en que el primer semestre de estudios es un punto determinante en la decisión de las mujeres de permanecer o abandonar la carrera (Miliszewska et al., 2006).

Entendiendo la importancia de incrementar la representación femenina en las carreras informáticas, se ha sugerido que acciones como los ambientes de aprendizaje colaborativo o la programación con pares pueden facilitar el éxito de estudiantes con menos experiencia en programación (Murphy et al., 2006). Más aún, algunos estudios han determinado que dichas técnicas contribuyen no solo a ayudar a las estudiantes, sino que también incrementan el éxito y reducen la deserción de estudiantes de ambos sexos (Berenson, Slaten, Williams y Ho, 2012; McDowell, Werner, Bullock y Fernald, 2003).

Otros autores como Rich et al. (2004) abogan por el diseño de cursos que aborden los estereotipos de la informática como asocial e inhóspita, para ello contextualizan la programación de manera que sea relevante y motivadora, a la vez que fomentan el trabajo colaborativo. Según Wilson (2006), la alta deserción en el primer año de carrera puede también ser abordada con diferentes tipos de actividades de aprendizaje, que proporcionen a las mujeres ejemplos y trabajos asociados con aplicaciones en el mundo real y a los hombres ejemplos y trabajos asociados con juegos.

\section{El contexto costarricense}

En el contexto de las universidades públicas costarricenses, algunos estudios (Marín, Barrantes, \& Chavarría, 2008; Mata, Quesada y Raventos, 2012; Ramírez y Rodríguez, 2013) han confirmado importantes diferencias en la participación de hombres y mujeres en las carreras del área informática. De acuerdo con Mata, Quesada et al. (2012), los programas de informática de estas universidades muestran una disminución notable en la presencia de mujeres a partir del año 2006 y de continuar la tendencia de perder un punto porcentual promedio de mujeres 
doi: http://dx.doi.org/10.15359/ree.21-1.12

URL: http://www.una.ac.cr/educare

CORREO: educare@una.cr

al año, estas se habrán extinguido en menos de 25 años. Esta situación es diferente a nivel de posgrado, como se documenta en Marín et al. (2008), ya que en este nivel no se evidencia una tasa descendiente en la admisión o reclutamiento de mujeres. Según Marín et al. (2008), la diferencia entre las mujeres y los hombres que se gradúan no se debe a situaciones de abandono o fracaso, sino que es un resultado del escaso número de mujeres que ingresan a estudiar la disciplina, y que a su vez esta última situación está vinculada a la naturaleza de los roles socialmente definidos. Un estudio más específico (Ramírez y Rodríguez, 2013) muestra que las estudiantes a nivel colegial demuestran más interés en carreras del área de salud, desconocen sus habilidades en las ingenierías y no conocen los beneficios de estudiar carreras en esta área. Estos resultados son compatibles con los hallazgos internacionales.

\section{Metodología}

\section{Selección de datos}

El estudio fue realizado con datos obtenidos de la Universidad Nacional de Costa Rica a solicitud de quienes presentan esta investigación, se utilizaron las bases de datos sobre el historial de notas proporcionadas por el Departamento de Registro, en un período de 8 años, comprendidos entre el año 2007 al 2014. De las bases de datos, se seleccionó únicamente la información de estudiantes de la carrera de Bachillerato en Ingeniería en Sistemas de Información de la Escuela de Informática y Computación de la Sede Central, con los atributos más relevantes para este estudio. Como resultado se obtuvo un repositorio, con información sobre las notas de estudiantes en los cursos de programación del plan de estudios, específicamente: EIF200 Fundamentos de Informática, EIF201 Programación I, EIF204 Programación II, EIF206 Programación III, EIF209 Programación IV, y EIF400 Paradigmas de Programación. Según el plan de estudios en los tres primeros cursos se incorporan los conocimientos propios de la programación orientada a objetos (POO) de manera incremental, en Fundamentos de Informática se introducen la algoritmia y los conceptos básicos de POO, en Programación I y Programación II se continúa profundizando en el tema de POO: relaciones, contenedores, implementación y optimización, en Programación III se trabaja con bases de datos y en Programación IV se introduce al ambiente Web. Finalmente, en Paradigmas de Programación se enfrenta al estudiantado con otros paradigmas de programación, como son programación lógica, funcional, etc.

La tabla de datos resultantes se compone de 10389 registros y 6 atributos, correspondiendo a un total de 2156 registros de mujeres y 8233 registros de hombres, los cuales corresponden a la matrícula de 2828 estudiantes: 601 son mujeres y 2227 son hombres. Esta tabla sirvió de base para las subsiguientes etapas del proceso de descubrimiento de conocimiento. La base de datos, así como sus tablas fueron construidas con el sistema gestor de base de datos PostgreSQL. 
doi: http://dx.doi.org/10.15359/ree.21-1.12

URL: http://www.una.ac.cr/educare

CORREO: educare@una.cr

Adicionalmente, utilizando estadística descriptiva se analizaron los datos proporcionados por la Oficina de Control de Admisiones y Registro con respecto al número de estudiantes que por año solicitan ingresar a la UNA, mediante la prueba de admisión y la cantidad de estudiantes que finalmente se admiten en la carrera.

\section{Procesamiento de los datos}

El objetivo de esta etapa es obtener datos limpios; datos sin valores nulos o anómalos que permitan garantizar la calidad de los resultados. Por medio de consultas SQL ad-hoc se analizó cuidadosamente la calidad de los datos contenidos en cada uno de los atributos de la tabla. Como resultado de esta etapa y con el fin de generar conocimiento acerca de los factores de estudio se seleccionaron de la tabla: el número de identificación de cada estudiante, el sexo, el código de ciclo lectivo, el código de la materia y la nota obtenida, como los datos más relevantes para el estudio.

\section{Etapa de transformación}

El objetivo de esta fase es transformar la fuente de datos en un conjunto listo para aplicar las diferentes técnicas de extracción de conocimiento. Se pasaron los valores continuos a valores discretos, considerando además el tratamiento de las notas RJ: retiro justificado, P: perdida, NSP: no se presentó, como valores nominales para no perder la información.

\section{Etapa de descubrimiento y evaluación}

El objetivo de esta etapa es detectar patrones insospechados y de interés en los datos, aplicando tareas de descubrimiento (minería de datos). Los resultados más relevantes de estas tareas se analizan en las siguientes secciones relacionándolos con las dimensiones de reclutamiento (admisión) y la permanencia o retención.

\section{Análisis de los datos}

\section{Proceso de admisión}

La Universidad Nacional lleva a cabo un proceso de admisión muy complejo, este contempla que el estudiantado postule su nombre para ser admitido, a la vez que eligen dos carreras en las que está interesado como primera y segunda opción. Luego debe realizar el examen de admisión, que es una prueba escrita. La nota de ingreso a la UNA se determina con el resultado de esta prueba y el promedio de notas de los últimos dos años de educación secundaria. Postulantes que obtienen 450 puntos o más, de los 900 posibles, en la nota de 
doi: http://dx.doi.org/10.15359/ree.21-1.12

URL: http://www.una.ac.cr/educare

ingreso quedan como elegibles para ingresar a la carrera seleccionada como primera opción. A su vez, cada carrera define el número de estudiantes que pueden ser admitidos por año, y a partir de este dato se acepta el estudiantado con mejor nota de ingreso. Este proceso se lleva a cabo aproximadamente durante 9 meses, en los cuales quien postula puede cambiar sus opciones de carrera, según sus intereses y oportunidades.

La Escuela de Informática de la UNA es la unidad académica que más estudiantes acepta cada año: alrededor de 300 estudiantes. Sin embargo, este número de ingreso puede variar, como se muestra en la Tabla 2, por razones diversas: presupuestarias, decisiones de las autoridades universitarias, decisión de estudiantes de no matricular, etc.

Para estudiar el proceso de admisión, en este artículo se consideró a estudiantes que solicitaron la carrera Ingeniería en Sistemas de Información como su primera opción de carrera, para analizar el proceso de términos de sexo, de no ser así, el proceso de análisis se complica muchísimo, pues se consideró que esta decisión no afectaría el análisis de los datos.

En la Tabla 1 se muestran los datos de estudiantes que realizan el examen de admisión de la UNA (postulantes) y finalmente estudiantes que lograron su admisión (Adm), se clasifican por sexo.

Tabla 1: Estudiantado postulante que realiza examen de admisión vs admitido, años 2007-2015 por sexo

\begin{tabular}{|c|c|c|c|c|c|c|c|}
\hline \multirow{2}{*}{ Año } & \multicolumn{3}{|c|}{ Mujeres } & \multicolumn{3}{|c|}{ Hombres } & \multirow{2}{*}{$\mathrm{P}(\mathrm{FM})$} \\
\hline & Realizan examen & Adm & Pf & Realizan examen & Adm & $\mathrm{Pm}$ & \\
\hline 2007 & 504 & 43 & $9 \%$ & 1674 & 188 & $11 \%$ & $30 \%$ \\
\hline 2008 & 452 & 62 & $14 \%$ & 1570 & 251 & $16 \%$ & $29 \%$ \\
\hline 2009 & 468 & 52 & $11 \%$ & 1492 & 246 & $16 \%$ & $31 \%$ \\
\hline 2010 & 433 & 50 & $12 \%$ & 1589 & 208 & $13 \%$ & $27 \%$ \\
\hline 2011 & 366 & 31 & $8 \%$ & 1550 & 154 & $10 \%$ & $24 \%$ \\
\hline 2012 & 329 & 45 & $14 \%$ & 1430 & 260 & $18 \%$ & $23 \%$ \\
\hline 2013 & 362 & 52 & $14 \%$ & 1391 & 241 & $17 \%$ & $26 \%$ \\
\hline 2014 & 344 & 59 & $17 \%$ & 1232 & 239 & $19 \%$ & $28 \%$ \\
\hline 2015 & 418 & 81 & $19 \%$ & 1470 & 299 & $20 \%$ & $28 \%$ \\
\hline PROM & 408 & 53 & $13 \%$ & 1489 & 232 & $16 \%$ & $27 \%$ \\
\hline
\end{tabular}

Nota: Elaboración propia. 
doi: http://dx.doi.org/10.15359/ree.21-1.12

URL: http://www.una.ac.cr/educare

CORREO: educare@una.cr

Además, se determina en la Tabla 1 el porcentaje de mujeres que ingresa a la carrera en relación con la cantidad de postulantes femeninas (Pf), y de igual manera para los hombres $(\mathrm{Pm})$; estos datos están representados en la Figura 1, que muestra la tendencia de ingreso, según postulación de hombres y mujeres. La columna $\mathrm{P}(\mathrm{FM})$ representa la proporción de las postulantes femeninas a la carrera con respecto a los postulantes masculinos.

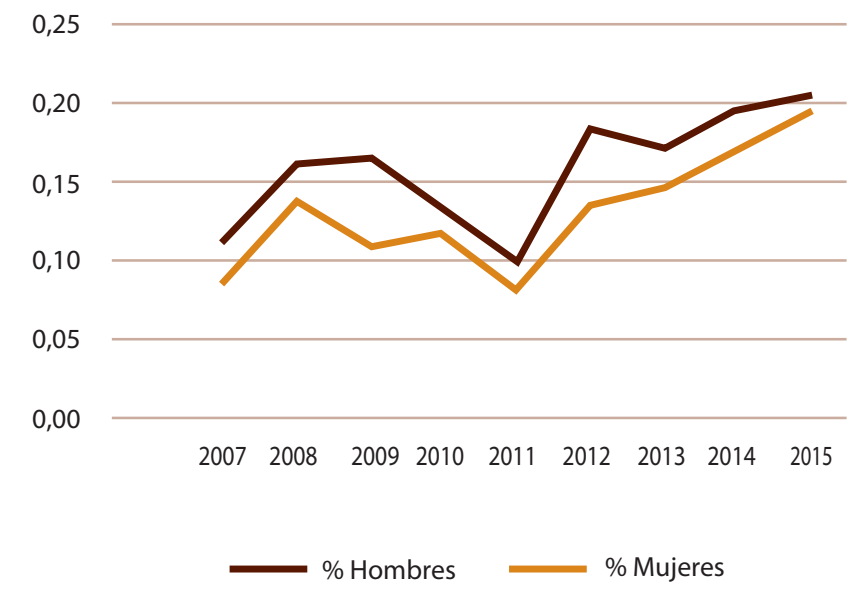

Figura 1: Estudiantado admitido vs postulante, años 2007-2014.

Como se puede observar en la Figura 1, el número de mujeres que se postula para la carrera es muy bajo con respecto a la cantidad de hombres que lo hacen, es un $27 \%$ en promedio, que prácticamente es una relación de 1 a 3 . Esto indica que la carrera despierta un interés mucho mayor en los hombres que en las mujeres, tal y como lo demuestran los estudios (Fisher et al., 1997; Margolis y Fisher, 2003; Scragg y Smith, 1998) y posiblemente sea necesario hacer un esfuerzo mucho mayor de promoción de la carrera a nivel de la educación secundaria, para incrementar la motivación hacia las carreras en esta área.

Puede verse también que el número de mujeres que finalmente entra a la carrera es muy bajo comparado con las mujeres que tenían la intención de hacerlo. Sin embargo, si analizamos la proporción femenina que ingresa (Pf) contra la proporción masculina que ingresa ( $\mathrm{Pm}$ ), mostrada en la Figura 1, puede verse que el comportamiento en este proceso es muy parecido para ambos. Las diferencias entre hombres y mujeres son en promedio de 3 puntos porcentuales, esto muestra que el porcentaje de hombres y mujeres que por distintas razones en el proceso desestiman la posibilidad de estudiar la carrera, ya sea por elección propia (son elegidos en otra universidad) o porque no obtienen los puntos requeridos para su admisión, es muy similar, es decir, no se puede argumentar que el proceso de admisión en sí mismo incida o repercuta en el bajo ingreso de las mujeres a la carrera. Lo anterior parece indicar que los bajos niveles de reclutamiento de mujeres no están asociados con el proceso de admisión que realiza la UNA. 
doi: http://dx.doi.org/10.15359/ree.21-1.12

URL: http://www.una.ac.cr/educare

CORREO: educare@una.cr

La Tabla 2 muestra el estudiantado admitido por sexo desde el año 2007 hasta el 2015:

Tabla 2: Admitidos a carrera. Años 2007-2015, por sexo

\begin{tabular}{ccccccc}
\hline \multirow{2}{*}{ Año } & $\mathrm{N}$ & \multicolumn{2}{c}{ Mujeres } & \multicolumn{2}{c}{ Hombres } & PFMadm \\
\cline { 3 - 6 } & & $\mathrm{Nm}$ & $\%$ & $\mathrm{Nh}$ & $\%$ & \\
\hline 2007 & 231 & 43 & 18,6 & 188 & 81,4 & $23 \%$ \\
2008 & 313 & 62 & 19,8 & 251 & 80,2 & $25 \%$ \\
2009 & 298 & 52 & 17,4 & 246 & 82,6 & $21 \%$ \\
2010 & 258 & 50 & 19,4 & 208 & 80,6 & $24 \%$ \\
2011 & 185 & 31 & 16,8 & 154 & 83,2 & $20 \%$ \\
2012 & 305 & 45 & 14,8 & 260 & 85,2 & $17 \%$ \\
2013 & 293 & 52 & 17,7 & 241 & 82,3 & $22 \%$ \\
2014 & 298 & 59 & 19,8 & 239 & 80,2 & $25 \%$ \\
2015 & 380 & 81 & 21,3 & 299 & 78,7 & $27 \%$ \\
\hline Prom & $\mathbf{2 8 4 , 5 6}$ & $\mathbf{5 2 , 7 8}$ & $\mathbf{1 8 , 4 0}$ & $\mathbf{2 3 1 , 7 8}$ & $\mathbf{8 1 , 6 0}$ & $\mathbf{2 3 \%}$ \\
\hline
\end{tabular}

Nota: Elaboración propia.

Los datos de la Tabla 2 muestran una desproporción entre la cantidad de mujeres y hombres que ingresan a la carrera de Ingeniería en Sistemas de Información. Puede verse que la proporción de ingreso de las mujeres con respecto a los hombres (PFMadm) es en promedio de un $23 \%$, y sustenta la diferencia de la representación femenina que se ha documentado ampliamente en numerosos estudios (Fisher et al., 1997; Lewis et al., 2007; Margolis y Fisher, 2003; Miliszewska et al., 2006; Scragg y Smith, 1998; Stoilescu y Egodawatte, 2010; Wilson, 2006).

Sin embargo, es importante destacar que el comportamiento de ingreso en el caso de la UNA, no muestra una tendencia a la baja como se documenta en otros estudios (Marín et al., 2008; Mata, Quesada et al., 2012), de hecho, a partir del año 2011 se muestra una sostenida tendencia al alza (Figura 2), asociado posiblemente al aumento en el número de estudiantado admitido. Se requiere un estudio futuro de corte más cualitativo para explicar esta situación y determinar acciones que permitan que el fenómeno se mantenga. 
doi: http://dx.doi.org/10.15359/ree.21-1.12

URL: http://www.una.ac.cr/educare

CORREO: educare@una.cr

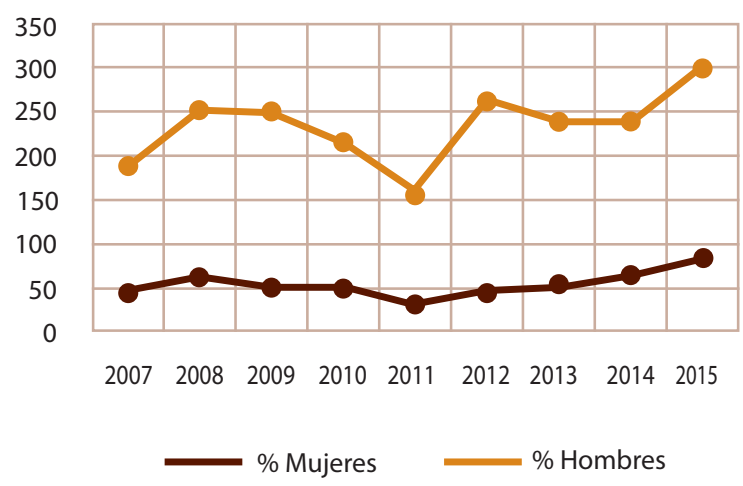

Figura 2: Estudiantado admitido a la carrera por sexo y por año, 2007-2015.

La Tabla 3 muestra el comportamiento en el caso del estudiantado graduado. En el período comprendido entre el 2007 y el 2014, el 74,6\% de los graduados son hombres y el 25,4\% son mujeres. Sin embargo, si consideramos que la admisión promedio de mujeres es únicamente del 18,4\% (Tabla 2), puede apreciarse que las mujeres son más efectivas para graduarse, sin embargo, esto queda aún más claro en las Tablas 3 y 4.

Tabla 3: Estudiantado graduado años 2007-2014 por sexo

\begin{tabular}{cccccc}
\hline \multirow{2}{*}{ Año } & \multicolumn{3}{c}{ Mujeres } & \multicolumn{2}{c}{ Hombres } \\
\cline { 2 - 6 } & $\mathrm{N}$ & $\mathrm{Nf}$ & $\%$ & $\mathrm{Nm}$ & $\%$ \\
\hline 2007 & 51 & 19 & 37,3 & 32 & 62,7 \\
2008 & 59 & 16 & 27,1 & 43 & 72,9 \\
2009 & 72 & 12 & 16,7 & 60 & 83,3 \\
2010 & 70 & 23 & 32,9 & 47 & 67,1 \\
2011 & 78 & 20 & 25,6 & 58 & 74,4 \\
2012 & 100 & 21 & 21,0 & 79 & 79,0 \\
2013 & 132 & 32 & 24,2 & 100 & 75,8 \\
2014 & 98 & 18 & 18,4 & 80 & 81,6 \\
\hline PROM & & & 25,4 & & 74,6 \\
\hline
\end{tabular}

Nota: Elaboración propia. 
doi: http://dx.doi.org/10.15359/ree.21-1.12

URL: http://www.una.ac.cr/educare

CORREO: educare@una.cr

Adicionalmente y bajo la premisa de que el promedio de graduación es de 5 años (obtenido a partir del estudio de graduación de 286 estudiantes, durante el período 2012-2015) se puede estimar el grado de efectividad al graduarse, como un parámetro a considerar. La Tabla 4 muestra los resultados obtenidos al comparar el estudiantado admitido en el 2007 con el graduado en el 2012 (en realidad terminan sus estudios en el 2011, pero se gradúan hasta el año siguiente), el admitido en el 2008 con el graduado en el 2013, y así sucesivamente.

Tabla 4: Porcentaje de efectividad en la carrera, por sexo

\begin{tabular}{ccc}
\hline Rango 5 años & Mujeres & Hombres \\
\hline $2007-2012$ & $49 \%$ & $42 \%$ \\
$2008-2013$ & $52 \%$ & $40 \%$ \\
$2009-2014$ & $35 \%$ & $33 \%$ \\
\hline
\end{tabular}

Nota: Elaboración propia.

En la Tabla 4 se hace más evidente que la proporción de las mujeres que se gradúan (dado el número de mujeres que ingresa) es mayor que la proporción de hombres que se gradúa según su ingreso, lo que es un indicador de que las mujeres son más efectivas en concluir el plan de estudios, tal y como lo mencionan (Byrne y Lyons, 2001; Marín et al., 2008; Murphy et al., 2006). Estos datos muestran que si se aumenta el número de mujeres que ingresan a la carrera es altamente probable que se aumente el número de estudiantado graduado en la disciplina, y este es un aspecto de amplio interés nacional, debido a las grandes demandas del sector TI en el país (Mata, Matarrita et al., 2012).

\section{Permanencia y retención}

El área de programación constituye un eje medular del plan de estudios, ya que se cuenta con seis cursos que se articulan a lo largo de los 4 años del plan. Además constituye, principalmente en sus dos primeros cursos, una de las áreas que mayor dificultad presenta al estudiantado, similar a lo documentado en investigaciones previas (Beyer et al., 2005, 2003; Margolis y Fisher, 2003; Scragg y Smith, 1998; Stoilescu y Egodawatte, 2010). Debido a lo anterior, se decidió analizar la permanencia de estudiantes en la carrera a partir de los datos de aprobación en los cursos del área de programación y de manera particular se analizó la deserción durante el primer año de carrera.

En laTabla 5 se muestran los porcentajes de aprobación generales en los cursos de programación. Como puede verse de los datos, con algunas excepciones, conforme se avanza en la carrera se mejora el índice de aprobación en los cursos, son preocupantes los índices de los dos primeros cursos (EIF200 y EIF201) que se imparten durante el primer año; sin embargo, se debe considerar que también el estudiantado en estos ciclos lleva otras materias como matemáticas, inglés y estudios generales. 
doi: http://dx.doi.org/10.15359/ree.21-1.12

URL: http://www.una.ac.cr/educare

CORREO: educare@una.cr

Tabla 5: Porcentaje de aprobación general, años 2007-2014 por curso

\begin{tabular}{ccccccc}
\hline Año & EIF200 & EIF201 & EIF204 & EIF206 & EIF209 & EIF400 \\
\hline 2007 & 52,4 & 60,5 & 53,4 & 66,3 & 85,1 & 86,4 \\
2008 & 44,4 & 66,8 & 64,2 & 71,0 & 76,9 & 75,6 \\
2009 & 50,5 & 59,7 & 66,5 & 91,4 & 77,9 & 68,8 \\
2010 & 42,0 & 49,3 & 69,6 & 89,2 & 79,2 & 85,3 \\
2011 & 50,7 & 60,1 & 59,4 & 79,1 & 80,9 & 63,6 \\
2012 & 52,3 & 61,9 & 76,4 & 66,3 & 57,6 & 61,0 \\
2013 & 50,5 & 62,4 & 65,1 & 64,0 & 82,3 & 55,2 \\
2014 & 48,0 & 51,5 & 60,6 & 57,8 & 65,7 & 70,1 \\
\hline PROM & 48,9 & 59,0 & 64,4 & 73,1 & 75,7 & 70,7 \\
\hline
\end{tabular}

Nota: Elaboración propia.

En la Tabla 6, se analizan estos datos considerando el comportamiento por sexo:

Tabla 6: Proporción de aprobación de mujeres/hombres según matrícula, por curso y sexo

\begin{tabular}{ccccccccccccc}
\hline \multirow{2}{*}{ Año } & \multicolumn{3}{c}{ EIF200 } & \multicolumn{2}{c}{ EIF201 } & \multicolumn{2}{c}{ EIF204 } & \multicolumn{2}{c}{ EIF206 } & \multicolumn{2}{c}{ EIF209 } & \multicolumn{2}{c}{ EIF400 } \\
\cline { 2 - 12 } & Muj & Hom & Muj & Hom & Muj & Hom & Muj & Hom & Muj & Hom & Muj & Hom \\
\hline 2007 & 56,7 & 51,0 & 53,3 & 63,0 & 53,5 & 53,4 & 77,8 & 62,2 & 83,3 & 85,3 & 81,3 & 87,5 \\
2008 & 44,0 & 44,6 & 67,3 & 66,7 & 76,6 & 60,8 & 65,4 & 72,4 & 81,3 & 75,0 & 81,5 & 72,9 \\
2009 & 51,6 & 50,1 & 64,5 & 58,1 & 64,7 & 66,9 & 91,7 & 91,3 & 81,5 & 76,9 & 52,0 & 73,8 \\
2010 & 29,6 & 45,1 & 63,0 & 46,7 & 72,7 & 68,6 & 92,5 & 88,2 & 65,5 & 83,2 & 86,0 & 85,0 \\
2011 & 46,7 & 52,0 & 52,6 & 62,2 & 67,5 & 57,2 & 63,6 & 84,2 & 83,3 & 80,0 & 59,5 & 65,0 \\
2012 & 48,5 & 53,1 & 56,8 & 63,0 & 80,5 & 75,4 & 61,9 & 67,6 & 54,5 & 58,2 & 62,5 & 60,7 \\
2013 & 43,0 & 52,2 & 51,1 & 65,0 & 62,5 & 65,5 & 60,0 & 64,8 & 82,9 & 82,1 & 50,0 & 56,3 \\
2014 & 52,1 & 47,1 & 51,7 & 51,5 & 68,8 & 59,1 & 70,6 & 55,2 & 73,7 & 64,6 & 74,1 & 69,3 \\
\hline PROM & 46,53 & 49,40 & 57,54 & 59,53 & 68,35 & 63,36 & 72,94 & 73,24 & 75,75 & 75,66 & 68,36 & 71,31 \\
\hline
\end{tabular}

Nota: Elaboración propia. 
doi: http://dx.doi.org/10.15359/ree.21-1.12

URL: http://www.una.ac.cr/educare

Los datos mostrados en la Tabla 6 representan las proporciones de mujeres y hombres que aprueban un curso en relación con las mujeres y hombres que lo matricularon, es decir, es una proporción de la aprobación de los cursos, según el sexo. Por ejemplo, en el año 2007 se matricularon 104 mujeres en el curso EIF200 y lo aprobaron 59 de ellas, lo que representa un 56,7.

Como puede verse, no hay una gran variabilidad en los resultados para hombres y mujeres; no obstante, estas últimas tienden, en promedio, a aprobar en menor proporción 4 de los 6 cursos de programación (EIF200, EIF201, EIF209 y EIF400). En el curso EIF204 el porcentaje de aprobación de las mujeres es mayor y en el curso EIF206 el comportamiento es similar para hombres y mujeres, como puede observarse más claramente en la Figura 3.

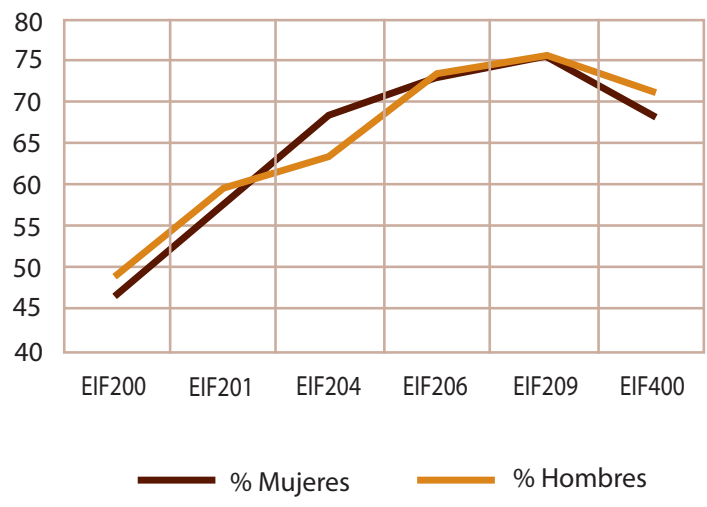

Figura 3: Promedio de aprobación de los cursos de programación años 2007-2014, por sexo.

Si se analiza el rendimiento académico, el promedio general de notas en los cursos de programación, es de un 67,4 para las mujeres y un 66,5 para los hombres. El desglose por curso puede verse en la Tabla 7, donde puede apreciarse, que a excepción del primer curso (EIF200), el promedio de notas de las mujeres es levemente mayor al de los hombres.

Tabla 7: Promedio general de notas, años 2007-2014 por sexo

\begin{tabular}{ccc}
\hline $\mathrm{N}$ & Mujeres & Hombres \\
\hline EIF200 & 57,5 & 57,7 \\
EIF201 & 62,5 & 61,3 \\
EIF204 & 65,6 & 65,1 \\
EIF206 & 71,0 & 71,3 \\
EIF209 & 74,7 & 72,8 \\
EIF400 & 73,3 & 70,7 \\
\hline PROM & 67,4 & 66,5 \\
\hline
\end{tabular}

Nota: Elaboración propia. 
doi: http://dx.doi.org/10.15359/ree.21-1.12

URL: http://www.una.ac.cr/educare

CORREO: educare@una.cr

En el tema de deserción y analizando los procesos correspondientes a lo largo de los cursos de programación, se obtiene la Figura 4 que muestra cómo la matrícula va disminuyendo conforme se avanza en el plan de estudios.

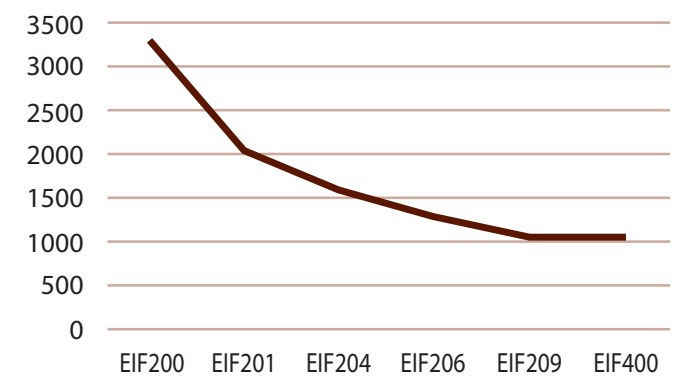

Figura 4: Matrícula cursos de programación, años 2007-2014.

El número de estudiantes que matriculan la secuencia de cursos de programación decrece particularmente después del primer curso EIF200 (lo que es un indicador de los niveles de deserción a lo largo de la carrera). Un aspecto positivo es que los índices de aprobación de los cursos hacen el proceso inverso, de esta manera, aunque el estudiantado es cada vez menos, un mayor número aprueba más los cursos conforme avanzan en el plan de estudios. Lo anterior explica también la poca cantidad de estudiantes que se gradúan, como vimos anteriormente.

A raíz de estos resultados, se decide analizar la deserción durante el primer año de estudios, por considerar que, en este, los cursos tienen índices de aprobación más bajos (particularmente las mujeres). Es de interés conocer cuántos estudiantes desisten de seguir estudiando la carrera, y ver si las mujeres se ven mayormente afectadas, como lo demuestran algunos estudios (Byrne y Lyons, 2001; Hagan \& Markham, 2000; Lewis et al., 2007; Margolis y Fisher, 2003; Sackrowitz y Parelius, 1996; Scragg y Smith, 1998; Wilson y Shrock, 2001). Para concluir que un estudiante abandona la carrera, se consideró como indicador el hecho de que no haya matriculado ningún curso desde el año 2012. En la Tabla 8 se presentan los resultados obtenidos:

Tabla 8: Datos acerca de deserción, años 2007-2014

\begin{tabular}{lcc}
\hline Estudiantado matriculado 2007-2014 & 2828 & \\
\hline Estudiantes que abandonan después del primer año & 810 & $29 \%$ \\
Hombres que abandonan después del primer año & 619 & $28 \%$ \\
Hombres que abandonan después del primer año sin haber perdido los cursos & 135 & $22 \%$ \\
Mujeres que abandonan después del primer año & 191 & $32 \%$ \\
Mujeres que abandonan después del primer año sin haber perdido los cursos & 42 & $22 \%$ \\
\hline
\end{tabular}

Nota: Elaboración propia. 
doi: http://dx.doi.org/10.15359/ree.21-1.12

URL: http://www.una.ac.cr/educare

De los 2828 estudiantes que se analizaron, 810 se retiraron de la carrera habiendo cursado el primer año de carrera, esto significa casi un 30\% del total de estudiantes en el período de estudio, de estos, 619 son hombres (76\%) y 191 son mujeres (24\%). Proporcionalmente, también se tiene que de la cantidad de hombres que ingresan un $28 \%$ abandona la carrera y de las mujeres que ingresan un 32\% lo hace, lo que muestra que además de los bajos niveles de ingreso de mujeres a la carrera, el problema de la baja representación se acentúa pues estas tienden a abandonarla en un mayor número.

Es interesante hacer notar que del estudiantado que se retira de la carrera en el primer año, 135 hombres y 42 mujeres lo hacen a pesar de no haber perdido nunca un curso de esta misma. Las causas más probables de este abandono están relacionadas al descubrimiento de que la carrera no es la adecuada a sus intereses.

Un elemento interesante que arrojaron los resultados, consistente con Wilson (2002), es que los hombres tienden a mostrar mayor perseverancia en la carrera, probablemente esto se explica porque tienen una motivación mayor para continuar, de ahí que matriculan más veces un curso, como puede verse en la Tabla 9, donde $\mathrm{N}$ es la cantidad de veces que matriculan el curso EIF200. Además, las mujeres tienden a desistir a partir de la 4 vez que llevan el curso, no así los hombres.

Tabla 9: Cantidad de veces que se matricula EIF200, años 2007-2014 por sexo

\begin{tabular}{rrr}
\hline$N$ & Mujeres & Hombres \\
\hline 1 & 350 & 1300 \\
2 & 109 & 423 \\
3 & 26 & 91 \\
4 & 4 & 24 \\
5 & 0 & 10 \\
6 & 1 & 2 \\
7 & 0 & 1 \\
\hline
\end{tabular}

Nota: Elaboración propia.

\section{Discusión}

Los datos muestran que el proceso de admisión a la Universidad no es excluyente, por lo que las razones de la marcada diferencia entre hombres y mujeres están probablemente fuera del contexto universitario, es muy posible que en la decisión de las últimas por la carrera que desean estudiar. En este sentido se deben reforzar las acciones que motiven a las estudiantes de 
doi: http://dx.doi.org/10.15359/ree.21-1.12

URL: http://www.una.ac.cr/educare

CORREO: educare@una.cr

secundaria en la elección de una carrera en el área de la informática, ya que, de no cambiar este panorama, a mediano plazo, la disciplina puede perder el aporte que el sexo femenino brinda en cuanto a habilidades y diferentes perspectivas.

Sin embargo, un resultado valioso es que, a diferencia de otros estudios (Marín et al., 2008; Mata, Quesada et al., 2012), en la UNA no se muestra en los últimos años una tendencia a la baja en el comportamiento de ingreso de las mujeres a la carrera, de hecho a partir del año 2011 se muestra una sostenida tendencia al alza. En este contexto es importante determinar acciones que permitan que el fenómeno se mantenga.

De los resultados se concluye también, que al igual que en otro estudio costarricense (Marín et al., 2008), las mujeres tienden a ser más efectivas al graduarse. Este dato es de particular importancia nacional, ya que actualmente existe en el país un déficit de profesionales en TI (Mata, Matarrita y Pinto, 2012), y de incrementar el número de ingresos femeninos a las carreras informáticas, es probable que se produzca como resultado un incremento en el número de estudiantado graduado y, por ende, un mayor número de profesionales en el área.

Con respecto a la primera interrogante planteada al inicio del artículo: ¿Cuál es la representación de la mujer en la carrera de Ingeniería en Sistemas de Información de la UNA?, podemos concluir que el ingreso de las mujeres a la carrera con relación al ingreso de los hombres se encuentra en el orden promedio de $23 \%$ mujeres $-77 \%$ hombres, lo que confirma la baja representación femenina en la carrera, tal y como se ha venido evidenciando mundialmente (Fisher et al., 1997; Lewis et al., 2007; Margolis y Fisher, 2003; Miliszewska et al., 2006; Scragg y Smith, 1998; Stoilescu y Egodawatte, 2010).

Con respecto a la segunda interrogante: ¿Cuáles son los índices de aprobación en el área de programación? ¿Muestran alguna diferencia los resultados de las mujeres con respecto a los hombres?, se desprende de los datos que no hay una gran variabilidad en los resultados para hombres y mujeres, sin embargo, estas últimas tienden, en promedio, a aprobar en menor proporción 4 de los 6 cursos de programación. Actualmente no tenemos datos que expliquen este comportamiento, ni su relación con otras variables como docentes, actividades de aprendizaje, tipo de evaluación, entre otros aspectos. Con respecto al rendimiento académico, el promedio general de notas en los cursos de programación de las mujeres es ligeramente superior.

Los datos confirman que el primer año de estudios es un año crítico que merece especial atención (Beyer et al., 2005; Goold y Rimmer, 2000; Miliszewska et al., 2006) lo que nos lleva a la tercera interrogante: ¿Qué impacto tienen los dos primeros cursos de programación en la permanencia de las mujeres en la carrera?

Del estudio se desprende que los dos primeros cursos de la carrera son especialmente críticos para la totalidad de estudiantes, pero que en promedio los hombres tienen un mejor desempeño que las mujeres. 
doi: http://dx.doi.org/10.15359/ree.21-1.12

URL: http://www.una.ac.cr/educare

CORREO: educare@una.cr

Con respecto a la permanencia, en el período estudiado, se retiraron de la carrera, después de su primer año, el 30\% de estudiantes, con una representación del $76 \%$ masculino y el $24 \%$ femenino, lo que es de esperar dada la proporción de ambos que se tiene al ingreso de la carrera. Proporcionalmente, la deserción representó un $28 \%$ de los hombres y un 32\% de las mujeres que ingresaron a la carrera, esto muestra que hay una mayor deserción de mujeres durante el primer año, lo que representa un factor más a considerar en el estudio de la baja representación femenina. Un dato interesante que mostró el estudio es que tanto el $22 \%$ de las mujeres como el $22 \%$ de los hombres que se retiran lo hacen sin haber perdido nunca ninguno de los dos cursos, lo que parece indicar que las causas están asociadas al descubrimiento de que la carrera no es la adecuada a sus intereses. Además, resultó interesante notar que los hombres tienden a mostrar mayor perseverancia en la carrera, lo que se evidencia en el número de veces que tienden a matricular un curso en contraste con las mujeres.

\section{Conclusiones}

Los resultados obtenidos permitieron confirmar la baja representación femenina en la carrera, pero parece no deberse a procesos propios de la admisión. Con respecto a los niveles de aprobación en los cursos de programación, se identificó que los resultados para hombres y mujeres son bastante similares, sin embargo, estas últimas tienden, en promedio, a aprobar en menor proporción los cursos de programación, pero su rendimiento académico en estos cursos tiende a ser ligeramente superior.

Un hallazgo, identificado previamente por otros estudios y confirmado por el nuestro, es que el primer año de la carrera es especialmente difícil para la totalidad de estudiantes, particularmente para las mujeres, las cuales muestran un mayor índice de deserción. El entender mejor esta situación brinda información importante para la toma de decisiones, no solo para mejorar las condiciones de las mujeres en la carrera, sino también para entender que se podrían tomar algunas decisiones que permitan aumentar el número de estudiantado graduado en el área, asunto que es de interés institucional, nacional e internacional.

\section{Trabajo futuro}

El estudio realizado representa el primer análisis de la situación de las mujeres en la carrera en la UNA. Se hacen necesarios estudios más integrales y complejos, que permitan analizar algunos otros elementos de corte más cualitativo, como la experiencia previa en programación, el concepto de auto-eficacia que tengan las mujeres y el posible impacto de roles docentes femeninos o masculinos, así como también el efecto de otros cursos complementarios que se llevan durante el primer año de la carrera como los cursos de matemática. 
doi: http://dx.doi.org/10.15359/ree.21-1.12

URL: http://www.una.ac.cr/educare

CORREO: educare@una.cr

Adicionalmente, se debe trabajar más en establecer estrategias que permitan a las mujeres un mayor ingreso a la carrera, así como una mayor permanencia en la misma. También, una mejor comprensión del fenómeno en el área de programación permitirá establecer estrategias pedagógicas que contribuyan no solo a ayudar a la permanencia de las mujeres sino que también permitirían incrementar el éxito y reducir la deserción de estudiantes de ambos sexos.

\section{Agradecimientos}

Agradecemos al Sr. Marvin Sánchez, Director del Departamento de Registro de la Universidad Nacional, y al profesor de la Escuela de Informática, Marco Zamora, la disposición y la ayuda brindada para obtener los datos utilizados en este artículo.

\section{Referencias}

Berenson, S. B., Slaten, K. M., Williams, L. y Ho, C.-W. (2012). Voices of women in a software engineering course: Reflections on collaboration. Journal on Educational Resources in Computing, 4(1). doi: https://doi.org/10.1145/1060071.1060074

Beyer, S., Dekeuster, M., Walter, K., Colar, M. y Holcomb, C. (2005). Changes in CS students'attitudes towards CS over time: An examination of gender differences. ACM SIGCSE Bulletin, 37(1), 392-396. doi: https://doi.org/10.1145/1047124.1047475

Beyer, S., Rynes, K., Perrault, J., Hay, K. y Haller, S. (2003). Gender differences in computer science students. ACM SIGCSE Bulletin, 35(1), 49-53. doi: https://doi.org/10.1145/792548.611930

Byrne, P. y Lyons, G. (2001). The effect of student attributes on success in programming. ACM SIGCSE Bulletin, 33(3), 49-52. doi: https://doi.org/10.1145/507758.377467

Cohoon, J. (2002). Recruiting and retaining women in undergraduate computing majors. ACM SIGCSE Bulletin, 34(2), 48-52. doi: https://doi.org/10.1145/543812.543829

Fisher, A., Margolis, J. y Miller, F. (1997). Undergraduate women in computer science: Experience, motivation and culture. ACM SIGCSE Bulletin, 29(1), 106-119 doi: https://doi. org/10.1145/268085.268127

Goold, A. y Rimmer, R. (2000). Factors affecting performance in first-year computing. ACM SIGCSE Bulletin, 32(2), 39-43. doi: https://doi.org/10.1145/355354.355369

Hagan, D. y Markham, S. (2000). Does it help to have some programming experience before beginning a computing degree program? ACM SIGCSE Bulletin, 32(3), 25-28. doi: https:// doi.org/10.1145/353519.343063 
doi: http://dx.doi.org/10.15359/ree.21-1.12

URL: http://www.una.ac.cr/educare

CORREO: educare@una.cr

Katz, S., Allbritton, D., Aronis, J., Wilson, C. y Soffa, M. L. (2006). Gender, achievement, and persistence in an undergraduate computer science program. ACM SIGMIS Bulletin, 37(4), 42-57. doi: https://doi.org/10.1145/1185335.1185344

Klawe, M. y Leveson, N. (1995). Women in computing: Where are we now? Communications of the ACM, 38(1), 29-35. doi: https://doi.org/10.1145/204865.204874

Lamas, M. (Comp.). (1997). El género: La construcción cultural de la diferencia sexual. México, DF MX: Miguel Angel Porrúa. Grupo Editorial.

Lewis, S., Lang, C. y McKay, J. (2007). An inconvenient truth:The invisibility of women in ICT.Australasian Journal of Information Systems, 15(1), 59-76. doi: https://doi.org/10.3127/ajis.v15i1.479

Margolis, J. y Fisher, A. (2003). Unlocking the clubhouse: Women in computing. Cambridge: Massachusetts Institute of Technology.

Marín, G., Barrantes, E. G. y Chavarría, S. (2008). Are women becoming extinct in the Computer Science and Informatics Program? CLEI Electronic Journal, 11(2), 1-11. Recuperado de http://www.clei.org/cleiej/papers/v11i2p6.pdf

Mata, F. J., Matarrita, R. y Pinto, C. (2012). Assessing computer education in Costa Rica: Results of a supply and demand study of ICT human resources. CLEI Electronical Journal, 15(1), 1-18. doi: https://doi.org/10.1109/CLEl.2012.6427245

Mata, F. J., Quesada, A. y Raventós, G. M. (2012). Gender gap in computer science programs from Costa Rican Public universities. En Informatica (CLEI), 38 Conferencia Latinoamericana (pp. 1-7). http://doi.org/10.1109/CLEl.2012.6427245

McDowell, C., Werner, L., Bullock, H. E. y Fernald, J. (2003). The impact of pair programming on student performance, perception and persistence. En 25 International Conference on software engineering (pp. 602-607). USA: IEEE Computer Society. doi: https://doi. org/10.1109/icse.2003.1201243

Miliszewska, I., Barker, G., Henderson, F.y Sztendur, E. (2006). The issue of gender equity in computer science--what students say. Journal of Information Technology Education, 5, 107-120.

Murphy, L., Richards, B., Mccauley, R., Morrison, B. B., Westbrook, S. y Fossum, T. (2006). Women catch up: Gender differences in learning programming concepts. ACM SIGCSE Bulletin, 38(1), 17-21. doi: https://doi.org/10.1145/1124706.1121350

Ramírez, W. M. y Rodríguez, I. (Octubre, 2013). ¿Por qué ingresan tan pocas mujeres a la carrera de Informática Empresarial del Recinto de Tacares de la Universidad de Costa Rica? Un enfoque de género. Ponencia presentada en la 39 Conferencia Latinoamericana de Informatica, Venezuela. 
doi: http://dx.doi.org/10.15359/ree.21-1.12

URL: http://www.una.ac.cr/educare

CORREO: educare@una.cr

Rich, L., Perry, H. y Guzdial, M. (2004). A CS1 course designed to address interests of women. ACM SIGCSE Bulletin, 36(1), 190-194. doi: https://doi.org/10.1145/1028174.971370

Sackrowitz, M. G. y Parelius, A. P. (1996). An unlevel playing field: Women in the introductory computer science courses. ACM SIGCSE Bulletin, 28(1), 37-41. doi: https://doi. org/10.1145/236462.236488

Scragg, G. y Smith, J. (1998). A study of barriers to women in undergraduate computer science. ACM SIGCSE Bulletin 30(1), 82-86. doi: https://doi.org/10.1145/274790.273167

Stoilescu, D. y Egodawatte, G. (2010). Gender differences in the use of computers, programming, and peer interactions in computer science classrooms. Computer Science Education, 20(4), 283-300. doi: https://doi.org/10.1080/08993408.2010.527691

Ventura, P. y Ramamurthy, B. (2004). Wanted: CS1 students, no experience required. ACM SIGCSE Bulletin, 36(1), 240-244. doi: https://doi.org/10.1145/1028174.971387

Vilner, T. y Zur, E. (2006). Once she makes it, she is there: Gender differences in computer science study. ACM SIGCSE Bulletin, 38(3), 227-231- doi: https://doi.org/10.1145/1140123.1140185

Wilson, B. C. (2002). A study of factors promoting success in computer science including gender differences. Computer Science Education, 12(1-2), 141-164. doi: https://doi.org/10.1076/ csed.12.1.141.8211

Wilson, B. C. (2006). Gender differences in types of assignments preferred: Implications for computer science instruction. Journal of Educational Computing Research, 34(3), 245-255. doi: https://doi.org/10.2190/7FLU-VKJL-86RM-5RQG

Wilson, B. C. y Shrock, S. (2001). Contributing to success in an introductory computer science course: A study of twelve factors. En H.Walker, R. McCauley, J.Gersting el. Russell (Chairmen), Proceedings of the $32^{\text {nd }}$ SIGCSE Technical Symposium on Computer Science Education (pp. 184-188). SIGCSE. New York: ACM. doi: https://doi.org/10.1145/366413.364581 